\title{
Erratum: Avalanches triggered by Kelvin-Helmholtz instability in a cylindrical plasma device [Phys. Rev. E 100, 033212 (2019)]
}

\author{
Y. Lang ${ }^{\oplus}$, Z. B. Guo, X. G. Wang, and B. Li
}

Q (Received 19 February 2020; accepted 20 February 2020; published 2 March 2020)

DOI: 10.1103/PhysRevE.101.039902

We have found that we used a wrong expression of classical diffusion coefficient in Eq. (9). The equation should be

$$
D_{\perp}=\chi_{\perp}=1.652 \times 10^{-5} \frac{n}{\mathrm{~cm}^{-3}}\left(\frac{T_{e}}{\mathrm{eV}}\right)^{-(1 / 2)}\left(\frac{B}{\mathrm{G}}\right)^{-2} \ln \Lambda_{0} \mathrm{~cm}^{2} / \mathrm{s} .
$$

Since $T_{e}$ is around $1 \mathrm{eV}$, our conclusions still hold. 